\title{
Thermopile infrared sensor with precisely patterned high-temperature resistant black absorber layer
}

\author{
A. Ihring ${ }^{\text {a) }}$, U. Dillner ${ }^{\text {a) }}$, E. Kessler ${ }^{\text {a) }}$, T. Hoyer $^{\text {b) }}$ \\ a) Institut für Photonische Technologien e.V. (IPHT), Albert-Einstein-Str. 9, 07745 Jena, Germany \\ b) Hermsdorfer Institut für Technische Keramik e.V. (HITK), Michael-Faraday-Str. 1, 07629 Hermsdorf, \\ Germany
}

\begin{abstract}
A thermopile infrared microsensor with a new broad band absorber layer is presented. Based on special carbon particles incorporated in a heteropolysiloxane nano composite matrix, which can be spin-coated on a wafer, the new absorber layer is high-temperature resistant up to $300{ }^{\circ} \mathrm{C}$ and can be patterned precisely by etching technologies. The sensor has a circular absorber area of $0.5 \mathrm{~mm}$ diameter. If nitrogen-backfilled, the sensor showed a responsivity and time constant of $280 \mathrm{~V} / \mathrm{W}$ and $43 \mathrm{~ms}$, respectively. A detectivity of about $7 \times 10^{8} \mathrm{cmHz}^{1 / 2} / \mathrm{W}$ was achieved.
\end{abstract}

\section{Introduction}

As thermal detectors thermopiles exhibit the key advantages of this class of detectors, which are simplicity and ruggedness of construction corresponding to low cost, a uniform broadband response over the IR spectrum and an operation without cooling. Additional benefits of thermopiles include the generation of an output voltage signal proportional to the incoming radiation, its high linearity over many orders of magnitude in incident infrared power and its insensitiveness to substrate temperature variations. Moreover, thermopiles require neither an electrical bias nor an optical chopper and do not generate $1 / \mathrm{f}$ noise. The absorber layer capturing the IR radiation and transforming it into heat, which generates a temperature gradient measured by the thermopile, is a crucial component in a thermopile infrared sensor. Its absorbance is proportional to the sensor responsivity and detectivity, respectively. Hence, it should be as high as possible, preferably nearly $100 \%$, over a wavelength range as broad as possible. This requirement is best met by colloidal metal black coatings (cf., e.g., [1] and the references therein). However, these coatings have some disadvantages including their fragility and low robustness to mechanical, thermal and chemical influences that lead to severe problems in their high-temperature stability and restrict applicable patterning technologies to evaporation through punched masks.

On the other hand, the metrological requirements of an increasing number of industrial applications concerning the automotive branch, energy management, aeronautical engineering and space technology tend towards operating temperatures exceeding $125^{\circ} \mathrm{C}$ (MIL specification). This has led to a growing interest to extend in particular the range of the operating temperature of thermopile IR sensors. Therefore, a high-temperature resistant version of the thermopile sensor TS-72 [2] and its successor TS-80 was developed by IPHT. In these sensors, multiple-beam interference layers are employed as absorber layers. These layers are high-temperature resistant and can be precisely patterned. However, due to the physics behind the absorption in these layers [3], there is no broad band behaviour but an absorbance peak, even though relatively broad, which can be tuned either to the first (3-5 $\mu \mathrm{m})$ or second (8-12 $\mu \mathrm{m})$ IR atmospheric window.

Here we report on a new broad band absorber layer, which is both high-temperature resistant and precisely patternable using microtechnology. Thus it combines the advantages of colloidal metal black coatings (broad band absorbance) and multiple-beam interference layers (high-temperature resistiveness and precise patternability). This makes the new absorber layer well-suited for the implementation in thermopile infrared microsensors. As an example a thermopile sensor TS-80 [4] with the conventional multiple-beam interference absorber layer being replaced by the new absorber layer is presented below.

\section{Sensor setup}

The TS-80 sensor setup is illustrated in Figs. 1 and 2. The key component of the sensor is a miniaturized multijunction thermopile made by MEMS technology on a silicon wafer. It consists of 80 radially arranged junction pairs formed by evaporated $n-\mathrm{Bi}_{1-\mathrm{x}} \mathrm{Sb}_{\mathrm{x}}(\mathrm{x}=0.87)$ and $\mathrm{p}$-Sb thin films. The sensor chip is 


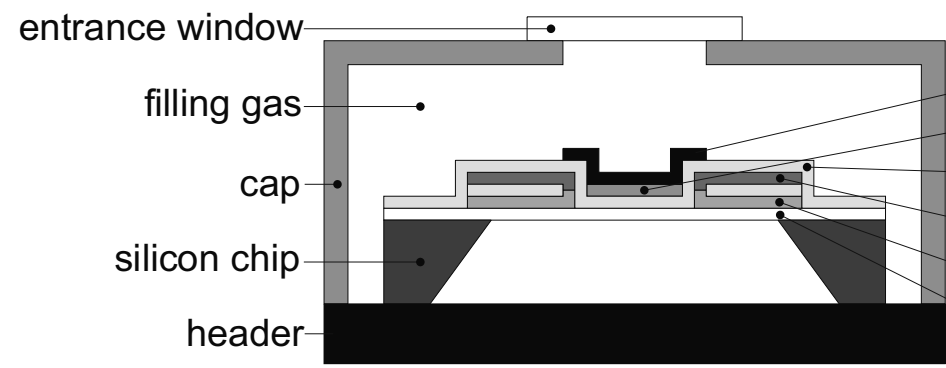

black absorber layer

thermal equalization layer

-isolation/passivation layer

p-type thermoelectric layer

n-type thermoelectric layer

supporting membrane

Fig. 1: Cross-sectional view of a thin-film thermopile IR sensor TS-80 showing the stack of the various functional layers including the broad band black absorber (schematic representation)

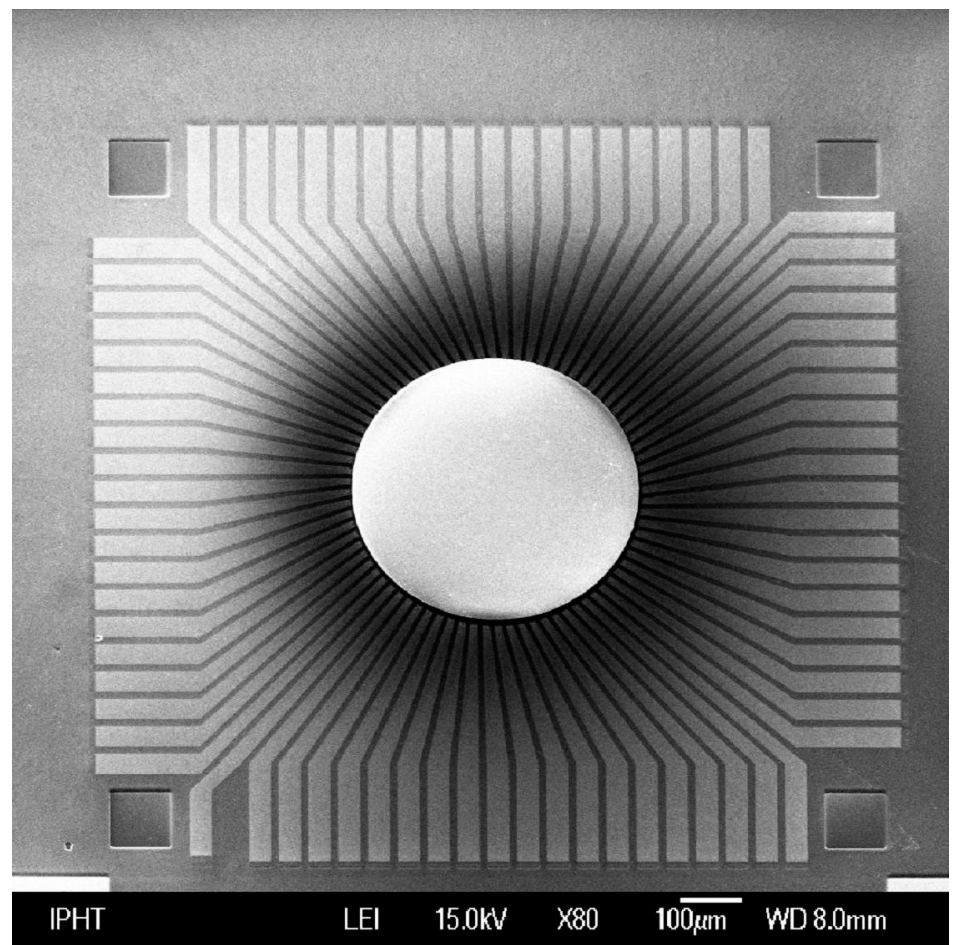

Fig. 2: Top view (scanning electron micrograph) of a chip of the thermopile IR sensor TS-80 with the high temperature resistant absorber layer (due to SEM imaging the absorber layer appears to be light contrary to optical imaging)

fabricated in a multi-layer technology [5], i.e., both legs of one thermocouple are arranged one upon the other with an isolating layer in between and are covered by a subsequent passivation layer (cf. Fig. 1).

\section{Broad band absorber layer}

The broad band absorber layer is based on special carbon particles incorporated in a heteropolysiloxane nano composite matrix, which can be spin-coated on a wafer. The nano composite is added for stabilizing the absorber layer. The patterning of these layers was realized by using a RIE technique (Reactive lon Etching). Therefore a structured aluminum layer on top was used as a mask. So a high selectivity was ensured between mask material and absorber layer during the patterning process. At first the upper thin nano composite layer was etched in a $\mathrm{SF}_{6}$ plasma for $10 \mathrm{~min}$. After this the thick absorber layer was etched in an $\mathrm{O}_{2}$ plasma. As final step the aluminum layer was removed by using a wet aluminum etch bath.

High patterning precision was achieved, which is demonstrated in Fig. 3. The new absorber layer is hightemperature resistant up to $300^{\circ} \mathrm{C}$. FTIR measurements of about $5 \mu \mathrm{m}$ thick samples of these layers revealed that, contrary to the multiple-beam interference layers mentioned above, they show a uniform broad band absorption in a wavenumber span from 5000 to $700 \mathrm{~cm}^{-1}$ (corresponding to a wavelength interval between 2 and $14 \mu \mathrm{m}$, cf. Fig. 4). 


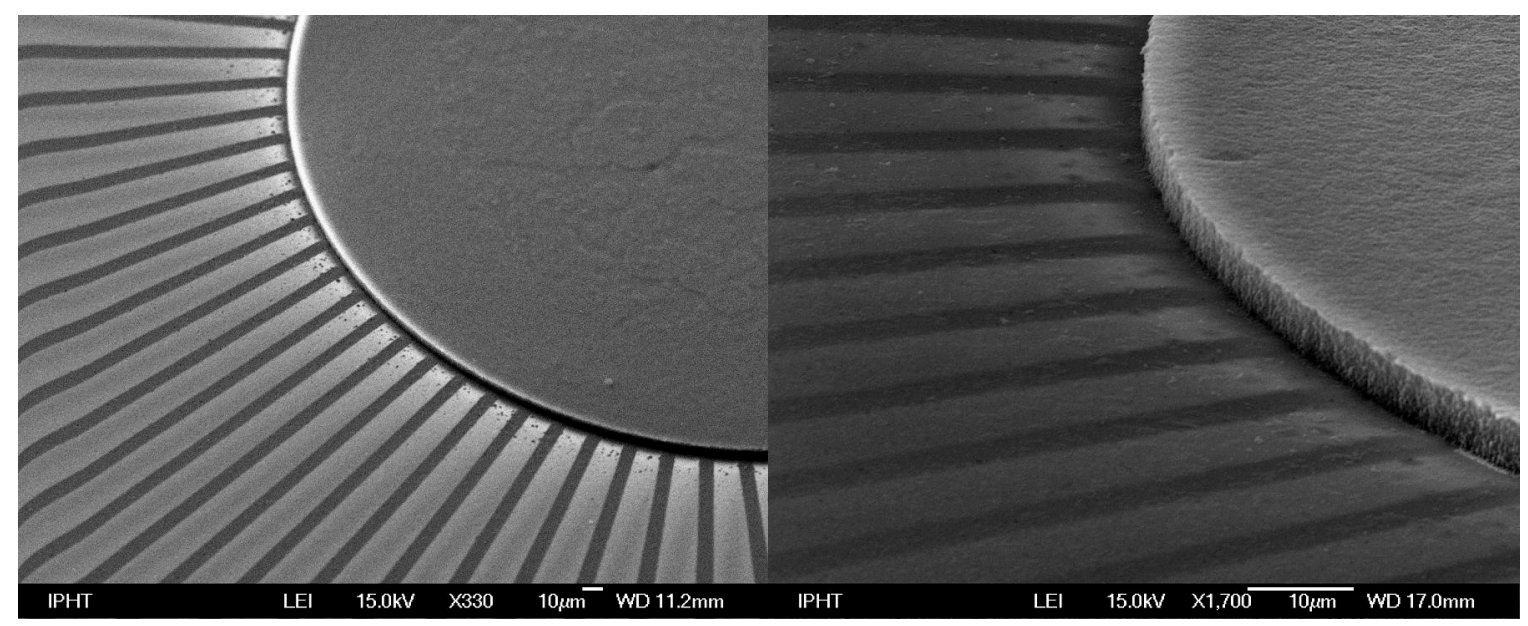

Fig. 3: Two scanning electron micrographs showing detailed views on the high temperature resistant black absorber layer illustrating the high precision of its patterning
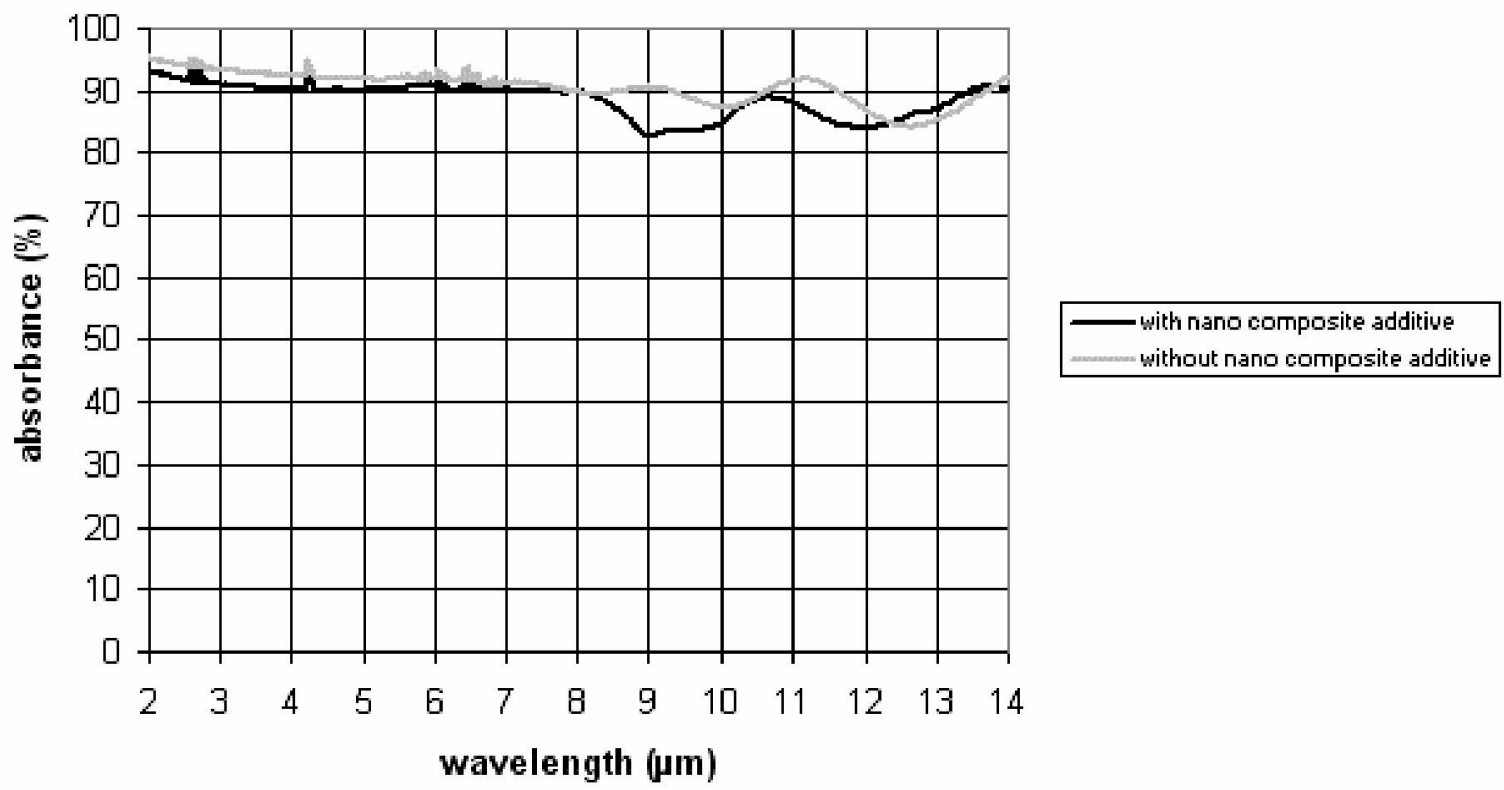

Fig. 4: Spectral absorbance of high temperature resistant black absorber layers in the IR wavelength range from 2 to $14 \mu \mathrm{m}$ (FTIR measurement)

\section{Sensor characterization}

Using the layer described above as absorber a high-temperature resistant broad-band IR sensitive variant of the TS-80 thermopile sensor has been realized. Its maximum operating temperature $\left(180{ }^{\circ} \mathrm{C}\right)$ is not limited by the absorber but by the thermopile layer materials. Measurements $(500 \mathrm{~K}$ black body radiation source, air environment corresponding to a nitrogen-backfilled sensor without filter) of the sensor performance data resulted in a responsivity and time constant of $280 \mathrm{~V} / \mathrm{W}$ and $43 \mathrm{~ms}$, respectively. The corresponding detectivity is about $7 \times 10^{8} \mathrm{cmHz}^{1 / 2} / \mathrm{W}$. Compared to a conventional thermopile sensor TS-80 of IPHT [4], both the sensor responsivity and time constant are raised by about $20 \%$. These experimental results are in accordance with prior FEM simulation calculations using ANSYS. The underlying FEM model is illustrated in Fig. 5.

A detailed characterization of the spectral transmittance $T$ and reflectance $R$ of the sensor membrane including both its absorber and thermopile regions was done using FTIR mapping. The results of a linear diagonal scan of the chip membrane is presented in Figs. 6 and 7 in a 3D spectral data representation. 
The capital letters in these two figures correspond to the following loci: $A=$ centre of the circular broad band black absorber, $\mathrm{B}=$ edge of the absorber layer, $\mathrm{C}=$ inner vertex of the membrane region not covered by the thermopile, $D=$ outer vertex of the membrane region not covered by the thermopile.

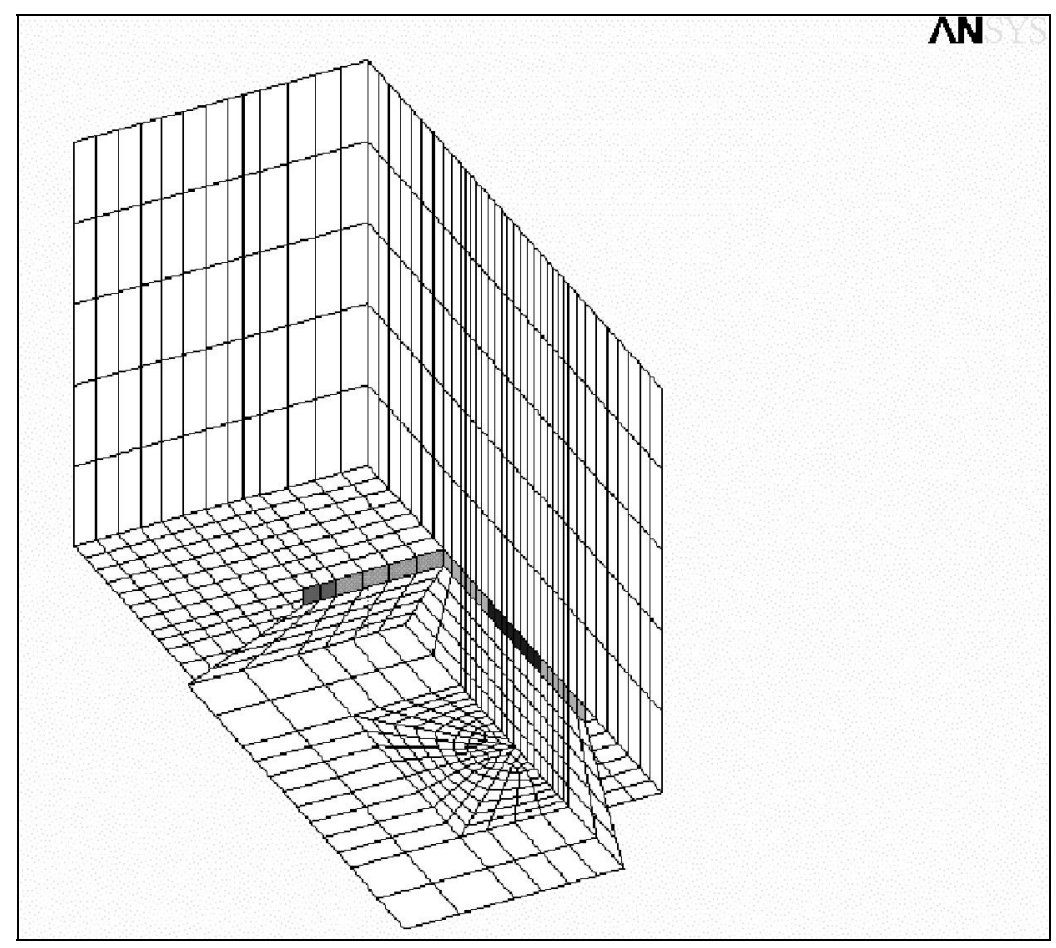

Fig. 5: 3D-FEM model of the TS-80 modeling region (light FE elements: filling gas, other FE elements: various membrane regions)

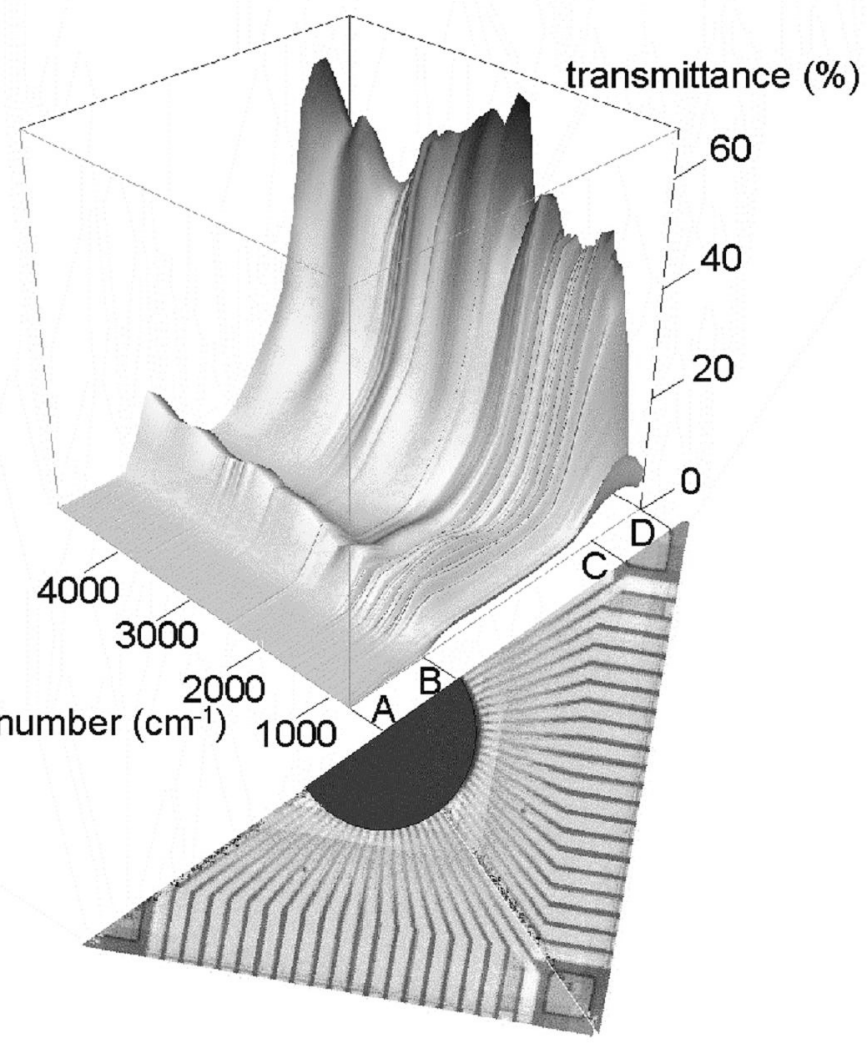

Fig. 6: Spectral transmittance of the half diagonal of a TS-80 sensor membrane in a wavenumber interval from 5000 to $700 \mathrm{~cm}^{-1}$ corresponding to 2 to $14 \mu \mathrm{m}$ wavelength (FTIR measurement) 


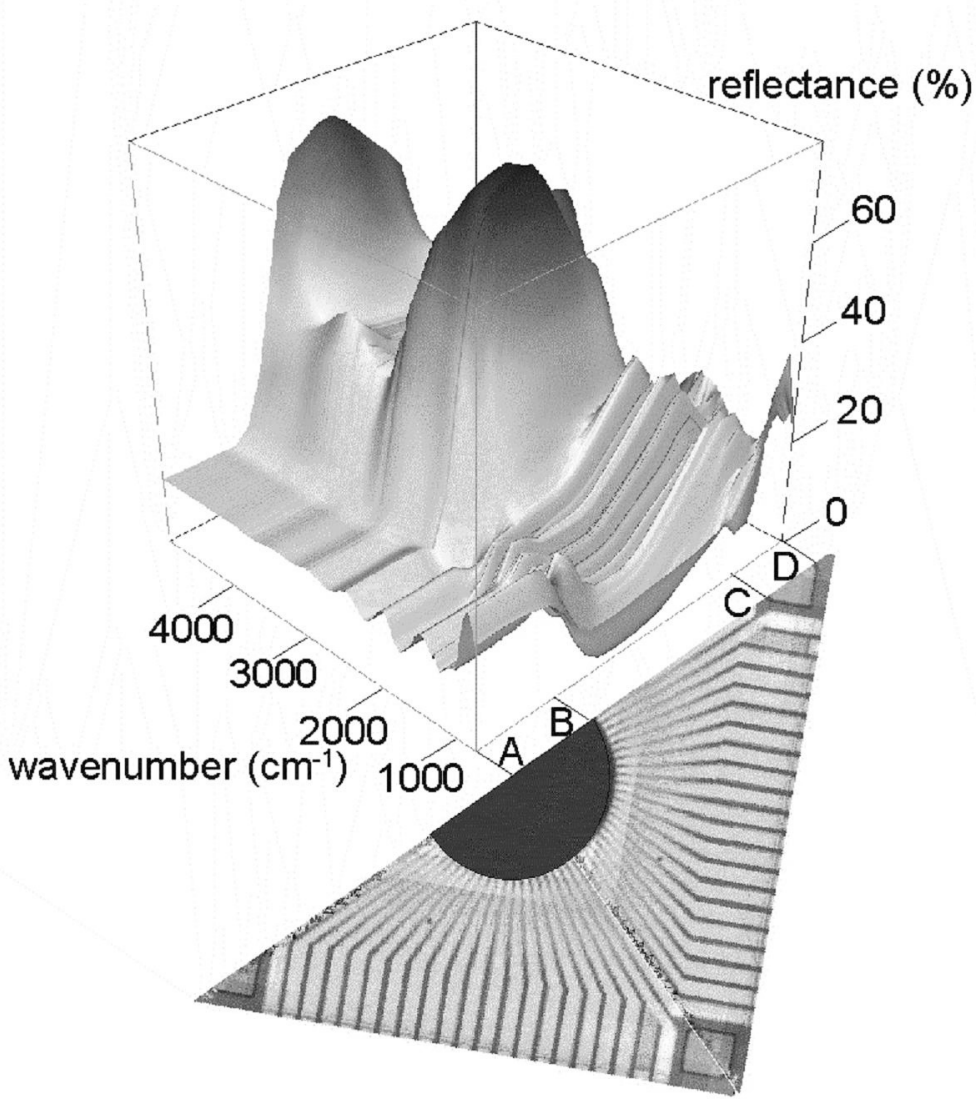

Fig. 7: Spectral reflectance of the half diagonal of a TS-80 sensor membrane in a wavenumber interval from 5000 to $700 \mathrm{~cm}^{-1}$ corresponding to 2 to $14 \mu \mathrm{m}$ wavelength (FTIR measurement)

Starting from $T$ and $R$ the spectral absorbance $A$ is defined by $A=1-T-R$. Fig. 6 illustrates that, as required, the region of the new broad band absorber between the locations $A$ and $B$ exhibits practically zero transmittance in the whole wavenumber interval considered contrary to the thermopile region between $B$ and $C$ where the gap between adjacent thermocouple legs causes a non-zero transmittance. Naturally, the membrane region between $C$ and $D$, which is not covered by any thermopile layers, shows the highest transmittance reaching about $60 \%$. As shown in Fig. 7, the reflectance of the broad band absorber is between about 10 and $20 \%$, which corresponds to an absorbance between about 80 and $90 \%$ according to the above definition. The spectral reflectance in the region covered by the thermopile layers is relatively high with maxima of approximately $70 \%$ due to the reflection of the semimetallic functional layers forming the thermocouples.

\section{Conclusion}

A high-temperature resistant and precisely patternable broad band absorber layer was presented. Replacing the conventional multiple-beam interference absorber layer, which features an absorbance peak typically either in the first $(3-5 \mu \mathrm{m})$ or the second $(8-12 \mu \mathrm{m}) \mathrm{IR}$ atmospheric window, by the new broad band $(2-14 \mu \mathrm{m})$ absorber layer in a standard thermopile sensor TS-80 of IPHT, the sensor responsivity and time constant are moderately modified, both increasing by about $20 \%$.

\section{Acknowledgment}

This work was funded by the Bundesministerium für Bildung und Forschung, project: fanimat nano layer, Grant No. 03WKF21G and 03WKF21A. 


\section{References}

[1] N. Nelms, J. Dowson, Goldblack coating for thermal infrared detectors, Sensors and Actuators A, 120 (2005) 403-407

[2] E. Kessler, V. Baier, U. Dillner, J. Müller, A. Berger, R. Gärtner, S. Meitzner, K.-P. Möllmann, Hightemperature resistant infrared sensing head, Proceedings of Sensor 2005, 12th International Conference, Vol. I, Nürnberg, 2005, pp. 73-78

[3] K.C. Liddiard, Application of interferometric enhancement to self-absorbing thin film thermal IR detectors, Infrared Phys., 34 (1993) 379-387

[4] Data sheet of TS-80, IPHT Jena, 2008

[5] F. Völklein, A. Wiegand; Sensors and Actuators A 24 (1990) 1-4 